\title{
Genetisch veränderte Viren gegen Krebs
}

Nachdem sich in einer Phase-I-Studie herausgestellt hatte, dass durch die Therapie mit dem onkolytischen Virus JX594 bei soliden Tumoren eine eine systemische Wirkung sowie ein komplettes Ansprechen erzielt werden können, prüften Onkologen die Wirksamkeit der intratumoralen Injektion des gentechnisch veränderten Vacciniavirus in niedrigen und hohen Dosierungen bei Patienten mit Leberkrebs. Von insgesamt 30 Patienten bekamen 16 eine hohe und 14 eine niedrige Virusdosis intratumoral injiziert. Die meisten Tumoren waren im fortgeschrittenen BCLC (Barcelona Clinic Liver Cancer)-Stadium C, also Stadium N1M1 plus Gefäßinvasion.

Das verwendete Virus wurde genetisch mit dem Gen für den Wachstumsfaktor GM-CSF sowie für das Enzym $\beta$-Galaktosidase ausgestattet. Der Wachstumsfaktor dient der Immunstimulation und das Gen für das Enzym als Marker für die Vermehrung des Vi- rus. Im Abstand von jeweils zwei Wochen wurde die Virussuspension innerhalb von vier Wochen dreimal intratumoral injiziert. Bereits 15 Minuten nach der Injektion tauchte das Virus in höchster Konzentration im Blut auf. Die Überlebenszeit war bei Patienten mit hoher Dosierung mit 14,1 Monaten signifikant höher als in der Vergleichsgruppe mit 6,7 Monaten (Hazard Ratio 0,39; $p=0,020$ ). Zum Vergleich: In einer aktuellen Phase-III-Studie mit Sorafenib lag die mediane Überlebensrate zwischen zwei und vier Monaten. Nach einem Jahr lebten noch $66 \%$ der Behandelten in der Hochdosisgruppe, in der Vergleichsgruppe waren es nur $23 \%$. Die Überlebensrate nach eineinhalb Jahren betrug 35 vs. $11 \%$.

Peter Leiner

Heo J et al. Randomized dose-finding clinical trial of oncolytic immunotherapeutic vaccinia JX594 in liver cancer. Nat Med. 2013 Feb 10. [Epub ahead of print]

\section{kurz notiert}

Mikro-RNAs als Ursache für Zweittumoren?

Die Haupttodesursache bei Darmkrebs sind Zweittumoren - ausgelöst von Krebszellen, die den Darm verlassen und sich meist in der Leber ansiedeln. Während Mikro-RNAs in gesunden Zellen eine Vielzahl lebenswichtiger Prozesse regeln, unterstützen manche MikroRNAs in Krebszellen offenbar das bösartige Wachstum. Beim Menschen sind bis heute über 700 verschiedene Mikro-RNAs entdeckt worden. „Wir vermuten, dass bestimmte Mikro-RNAs Krebszellen so umprogrammieren können, dass sich diese im Körper verbreiten", erklärt Heike Allgayer vom Deutschen Krebsforschungszentrum in Heidelberg.

Bei der Untersuchung von Patientengewebe entdeckten sie und ihr Team, dass bestimmte Mikro-RNAs in unterschiedlichen Mengen in gesundem Darmgewebe, Tumorgewebe und umherwandernden Krebszellen vorhanden sind. "Mikro-RNAs könnten bspw. die Produktion eines krebshemmenden Stoffes hemmen, der eigentlich das Auswandern in andere Organe verhindert", so Allgayer weiter. Die Heidelberger Forscher untersuchen nun, welche Prozesse von den verdächtigen Mikro-RNAs in Darmkrebszellen reguliert werden.

\section{Angst vor Lymphödem meist unbegründet}

Sarah A. McLaughlin und Kollegen beobachteten 120 Brustkrebspatientinnen über ein Jahr nach. Bei 53 von ihnen war eine axilläre Lymphknotendissektion (ALND), bei 67 nur eine Entfernung des Sentinellymphknotens (SLNB) erfolgt. Nach einem Jahr hatten signifikant weniger SLNB-Patientinnen ein Lymphödem entwickelt als Patientinnen mit ALND (3 vs. 19\%). Die Sorgen der Patientinnen entsprachen dieser Realität nicht: $75 \%$ der ALND- und 52\% der SLNB-Patientinnen befürchteten sechs Monate nach dem Eingriff ein Lymphom. Ein Schwellungsgefühl am Arm gaben nach sechs Monaten $25 \%$ der ALND- und $11 \%$ der SLNB-Patientinnen an, nach einem Jahr 31 bzw. $6 \%$. Also deutlich mehr als der Anteil der Patientinnen mit nachgewiesenem Lymphödem. Auch die Entscheidung der Patientin für eine Präventionsmaßnahme richtete sich wohl mehr nach dem Gefühl als nach der objektiven Messung.
Bei den meisten Patientinnen schien das Armvolumen zudem unabhängig von der Lymphomentwicklung zu schwanken. 114 Teilnehmerinnen hatten nach sechs Monaten ein um bis zu $9 \%$ gegenüber dem Ausgangswert verändertes Armvolumen, das sich unabhängig vom Tragen eines Kompressionsarmstrumpfs bei jeder dritten Betroffenen im nächsten halben Jahr wieder zurückbildete. In der Studie war nur das Ausmaß der Axilladissektion ein signifikanter Risikofaktor für die Entwicklung eines Lymphödems. Alter, BMI, Operationsart, Infektion oder Bestrahlung waren nicht mit einem erhöhten Risiko assoziiert. Fazit: Grundlage für die Beratung von Brustkrebspatientinnen nach Wächterlymphknotenbiopsie bilden immer noch Risikofaktoren wie BMI, Alter, Trauma oder Infektion, die sich jedoch nach McLaughlin et al. als wenig verlässlich erwiesen. Den Armumfang zu messen, bleibt unverzichtbar. Die Messungen

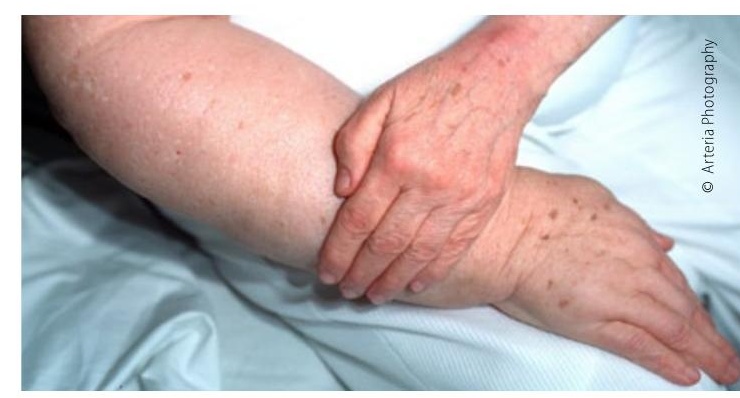

Viele Brustkrebspatientinnen befürchten, postoperativ ein Lymphödem zu entwickeln.

sollten langfristig fortgesetzt werden, um ein Lymphödem zu bestätigen. Laut den Forschern sollte die Patientenwahrnehmung als klinisch bedeutsamer Faktor anerkannt werden. Eine verbesserte Risikostratifizierung, z.B. mithilfe des von Bevilacqua entwickelten Nomogramms, könne helfen, unbegründete Ängste zu zerstreuen.

Elke Oberhofer

McLaughlin SA et al. Trends in Risk Reduction Practices for the Prevention of Lymphedema in the First 12 Months after Breast Cancer Surgery. J Am Coll Surg. 2013;216(3):380-9. 